\title{
FLT3 Internal Tandem Duplication
}

National Cancer Institute

\section{Source}

National Cancer Institute. FLT3 Internal Tandem Duplication. NCI Thesaurus. Code C67494.

A genetic abnormality that arises from duplications of the juxtamembrane portion of the gene and results in constitutive activation of the FLT 3 receptor tyrosine kinase protein in early hematopoietic progenitor cells. It is associated with acute myelogenous leukemia where it appears to correlate with a poor prognosis. 